\title{
The storm time central plasma sheet
}

\author{
R. Schödel ${ }^{1}$, K. Dierschke ${ }^{1}$, W. Baumjohann ${ }^{2}$, R. Nakamura ${ }^{2}$, and T. Mukai ${ }^{3}$ \\ ${ }^{1}$ Max-Planck-Institut für extraterrestrische Physik, Garching, Germany \\ ${ }^{2}$ Institut für Weltraumforschung der Österreichischen Akademie der Wissenschaften, Graz, Austria \\ ${ }^{3}$ Institute of Space and Astronautical Science, Sagamihara, Japan
}

Received: 28 January 2002 - Revised: 17 May 2002 - Accepted: 20 June 2002

\begin{abstract}
The plasma sheet plays a key role during magnetic storms because it is the bottleneck through which large amounts of magnetic flux that have been eroded from the dayside magnetopause have to be returned to the dayside magnetosphere. Using about five years of Geotail data we studied the average properties of the near- and midtail central plasma sheet (CPS) in the 10-30 $R_{E}$ range during magnetic storms. The earthward flux transport rate is greatly enhanced during the storm main phase, but shows a significant earthward decrease. Hence, since the magnetic flux cannot be circulated at a sufficient rate, this leads to an average dipolarization of the central plasma sheet. An increase of the specific entropy of the CPS ion population by a factor of about two during the storm main phase provides evidence for nonadiabatic heating processes. The direction of flux transport during the main phase is consistent with the possible formation of a near-Earth neutral line beyond $\sim 20 R_{E}$.
\end{abstract}

Key words. Magnetospheric physics (plasma convection; plasma sheet; storms and substorms)

\section{Introduction}

Magnetic storms are basically caused by prolonged periods of southward IMF that lead to greatly enhanced reconnection at the Earth's dayside magnetosphere and to a massive accumulation of magnetic flux in the tail lobes. This flux has to be returned to the dayside magnetosphere through the plasma sheet. It is the dynamical processes in the plasma sheet during the storm main phase, like substorm activity and convection, that are most likely responsible for the injection of energetic ions into the ring current and the depression of the $D_{s t}$ index. The question whether the frequent occurrence of magnetospheric substorms or whether enhanced magnetospheric convection alone is responsible for the accumulation of energetic particles in the near-Earth magnetosphere is still subject of considerable debate (Kamide et al., 1998).

Correspondence to: R. Schödel (rainer@mpe.mpg.de)
Recently, Lui et al. (2001) presented evidence that both processes are involved in causing the $D_{s t}$ depression observed during magnetic storms.

Baumjohann et al. (1996) compared the behavior of the near-Earth tail during storm-time and non-storm substorms, deriving the following picture. Substorms that occur during magnetic storms are accompanied by a much stronger dipolarization of the magnetic field in the central plasma sheet (CPS) than non-storm substorms. Parallel to the strong CPS dipolarization the lobe magnetic field pressure decreases during storm-time substorms, while it stays approximately constant during non-storm substorms. While both types of substorms heat the CPS ion population by about equal amounts, the ions in the storm time CPS have already a temperature about twice as high as the non-storm CPS ions before substorm onset. Baumjohann et al. (1996) concluded that during storm-time susbtorms lobe magnetic flux might be reconnected at a near-Earth neutral line (NENL), and that the stronger dipolarization and the higher temperature of the plasma sheet during storm-time substorms lead to an effective injection of energetic particles into the ring current.

In the last decade the Geotail satellite has provided extensive measurements of the magnetotail, especially from the near- and midtail regions. We examined $\sim 5$ years of Geotail data in order to address the question of the average behavior of the CPS in the 10-30 $R_{E}$ region during magnetic storms using $D_{s t}$ values for selecting the data according to magnetospheric activity.

\section{Data}

As a data base we used Geotail measurements from the region $-10 \geq X_{\mathrm{AGSM}} \geq-31 R_{E}$ obtained in the time between October 1994 and May 1999 (AGSM is the geocentric solar magnetospheric coordinate system (GSM) with an average tail aberration of $4^{\circ}$ ). The data set consists of 12 -s averages of plasma moments and magnetic field measurements. Plasma moments were measured by the low energy particle 
Table 1. Approximate numbers of Geotail 12-sec CPS samples for different levels of magnetospheric activity

\begin{tabular}{lll}
\hline Activity & $10-20 R_{E}$ & $20-30 R_{E}$ \\
\hline main phase & 5,200 & 3,800 \\
recovery phase & 23,100 & 32,000 \\
quiet times & 288,000 & 471,000 \\
\hline
\end{tabular}

Table 2. Average $D_{s t}$ values for the data in each distance bin

\begin{tabular}{lll}
\hline Activity & $10-20 R_{E}$ & $20-30 R_{E}$ \\
\hline main phase & -91 & -78 \\
recovery phase & -50 & -29 \\
quiet times & -8 & -7 \\
\hline
\end{tabular}

(LEP) experiment (Mukai et al., 1994) and the magnetic field data were obtained by the magnetic field (MGF) instrument (Kokubun et al., 1994).

Hourly final $D_{s t}$ indices for the corresponding period are provided by the World Data Center for Geomagnetism, Kyoto. From these $D_{s t}$ data we selected 81 storms that showed clear signatures of main and recovery phase. We defined the beginning of the main phase by the time when the $D_{s t}$ index started to drop toward the main phase minimum. Storm sudden comencement is not a useful marker because this feature cannot be clearly distinguished for many storms. We defined the end of the main phase and the beginning of the recovery phase by the time when $D_{s t}$ reached its peak (i.e., minimum) value. The end of the recovery phase was chosen as the time when $D_{s t}$ had decayed to $\sim 20 \%$ of its peak value. Quiet times were defined by $D_{s t}$ values $>-25$. In order to have sufficient samples for a statistical analysis, we chose to bin the data into only two radial distance ranges from 10$20 R_{E}$ and from $20-30 R_{E}$. Table 1 gives the number of CPS samples (12-s averages) for the main and recovery phases of magnetic storms, and for quiet times. The average $D_{s t}$ values per bin are indicated in Table 2.

The criterion for selecting samples from the CPS was $\beta>0.5$ (Angelopoulos et al., 1994). In order to assess the influence of this selection criterion on the data, we repeated our analysis for $\beta>0.1$ as well as for $\beta>1$. All of the examined parameters change by less than $20 \%$ when $\beta$ is varied over an order of magnitude. The advantage of our selection criterion is that it allows for an unbiased examination of the field elevation in the CPS.

\section{Properties of the storm-time CPS}

Based on our data selection as described above, we calculated average values of the magnetic field and of the plasma parameters in the CPS for the storm time main and recovery phases, as well as for quiet times. The characteristic values for the magnetic field strength, the magnetic field elevation angle, the ion temperature, and the specific entropy are displayed in Fig. 1. Values for the recovery phase lie generally between those for the main phase and those for quiet times, being usually not significantly different from the latter. Hence the CPS recovers within a fairly short time from the effects of a magnetic storm, with the exception of ion temperature, which remains significantly higher during the recovery phase than during quiet times.

During the main phase the magnetic field strength is raised by a factor of about two over the values at quiet times. The magnetic field elevation angle is about $30^{\circ}$ and approximately constant out to distances of $20-30 R_{E}$, and is thus considerably increased compared to quiet times. Consequently, on average, the CPS seems to be inflated in the region 10-30 $R_{E}$ during the storm main phase. Baumjohann et al. (1996) found that the magnetic field elevation in the 10-20 $R_{E}$ CPS is considerably increased by substorms that occur during storm times, while the lobe magnetic pressure decreases. These findings correspond well to the interpretation of an average dipolarization of the $10-30 R_{E}$ CPS during the storm main phase caused by a successive occurrence of substorms.

Like Baumjohann et al. (1996) we find that the average temperature of ions in the CPS is strongly increased during magnetic storms. Since the ion temperature remains significantly above quiet time levels even during the recovery phase, we conclude that the energetic ions remain trapped in the CPS and loose their energy only gradually, comparable to the slow, exponential decay of the ring current during the recovery phase.

Our definition of specific entropy, $k T / n^{2 / 3}$, is based on using a polytropic law of the form $P=\alpha N^{\gamma}$, with $\gamma=5 / 3$ (adiabatic case). $\alpha$ is a constant that depends on the specific entropy of the plasma (see Siscoe, 1983; Baumjohann, 1993). Thereby we assume that the majority of the density-pressure changes in the CPS follow an adiabatic path and that different plasma regimes such as high-speed flows and quiet plasma are characterised by different levels of their specific entropy. For an ample discussion on the issue see Baumjohann (1993). The increase of the specific entropy of the ions by a factor of two during the main phase indicates that they are heated by non-adiabatic processes. Liu and Rostoker (1995) have suggested such a non-adiabatic mechanism: They showed that the tail plasma will gain energy through non-adiabatic pitch angle scattering combined with Fermi and betatron accelerations if it is circulated recurrently between the tail and the inner magnetosphere by repeated substorm activity.

\section{Transport processes}

The increased ion bulk velocity and the greater average rate of earthward transport of magnetic flux density, $V_{X} B_{Z}$, reflect the enhanced circulation of plasma and magnetic flux through the magnetosphere during the storm main phase 

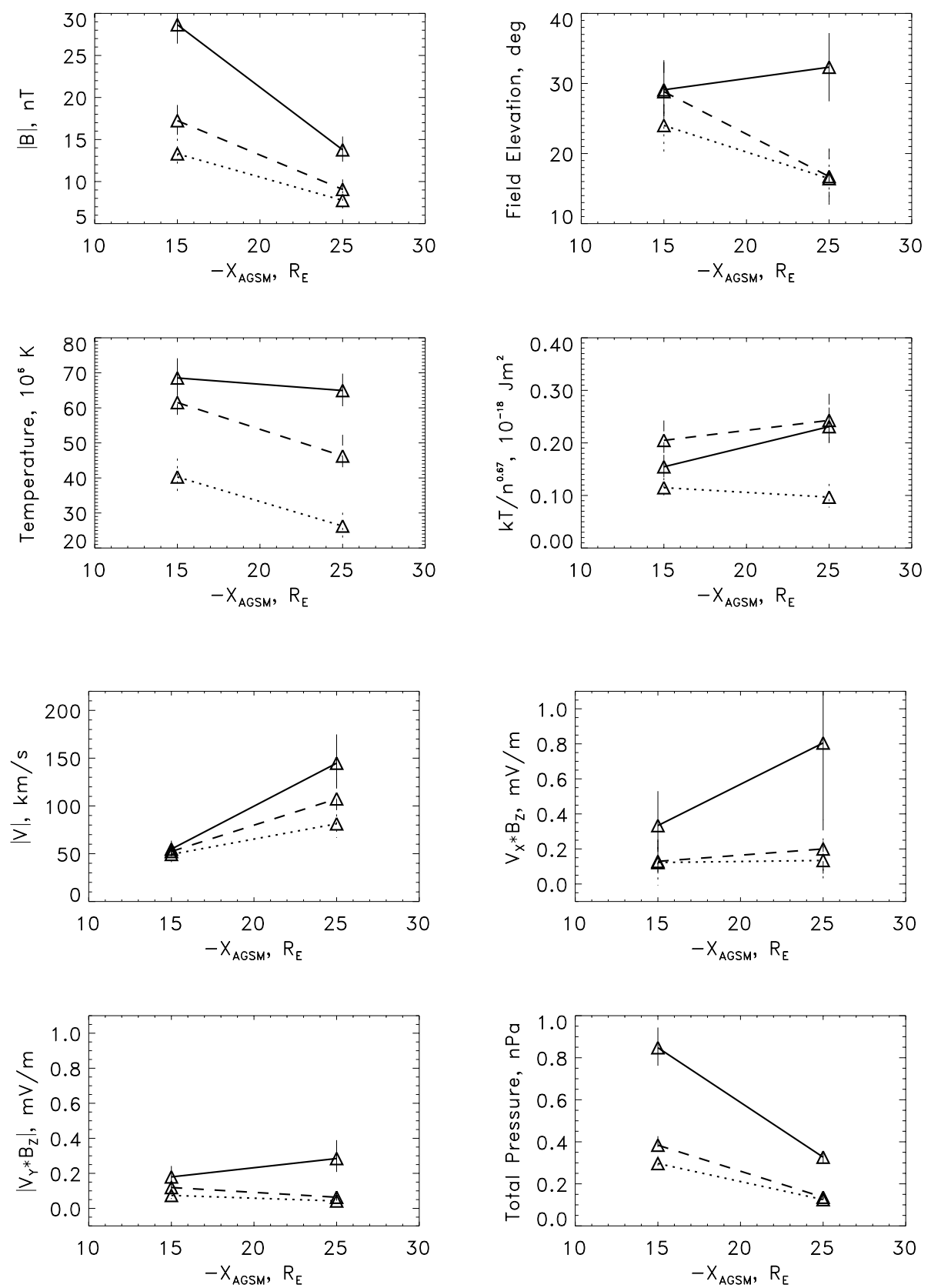
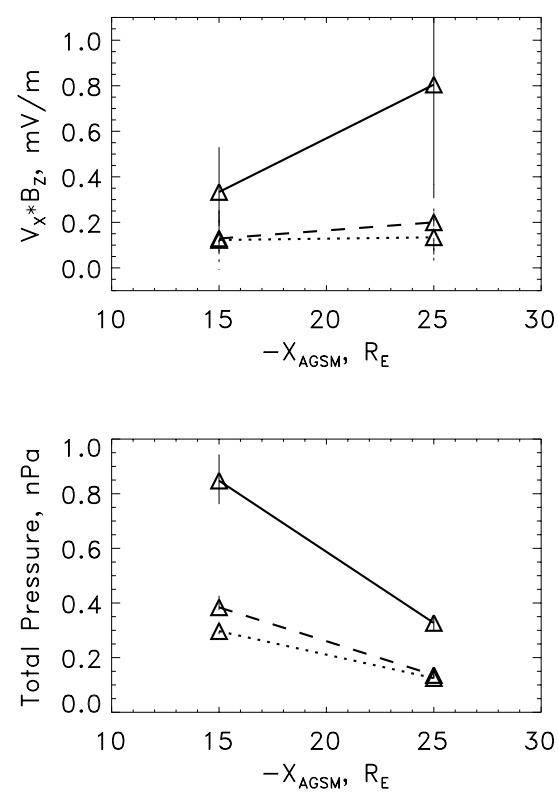

Fig. 1. Average values of the magnetic field strength, of the magnetic field elevation, of the total (i.e. thermal and magnetic) pressure, and of the ion specific entropy in the central plasma sheet. Solid lines: main phase, dashed: recovery phase, dotted: quiet times $\left(D_{s t}>\right.$ $-25)$. Error bars are $0.2 \sigma$ of the data distribution functions.
Fig. 2. Average values of the ion bulk velocity, of the rate of earthward transport of magnetic flux, $V_{X} B_{Z}$, of the overall level of east-westward transport of magnetic flux, $\left|V_{Y} B_{Z}\right|$, and of the total pressure in the central plasma sheet. Solid lines: main phase, dashed: recovery phase, dotted: quiet times $\left(D_{s t}>\right.$ $-25)$. Error bars are $0.2 \sigma$ of the data distribution functions.
(Fig. 2). However, a strong earthward decrease of the transport rates during the storm main phase is evident from the data. An explanation for this behavior may be the steep gradient of the total (i.e., thermal and magnetic) pressure during the main phase (Fig. 2). In order to check on the question whether the flux transport might be significantly diverted to eastward or westward directions during the main phase closer to Earth, we examined the average of $\left|V_{Y} B_{Z}\right|$ as well. One can see in Fig. 2 that the earthward decrease of $V_{X} B_{Z}$ cannot be explained by a diversion of the flux transport. Hence, assuming that the CPS does not expand in the earthward direction, we can conclude that during the storm main phase magnetic flux cannot be returned at a sufficient rate toward the dayside magnetosphere in order to maintain a steady average transport rate. Since the magnetic field elevation is increased during the main phase as mentioned in the previous section, we favour the assumption that the hindered transport processes in the plasma sheet lead to a dipolarization which is rapidly progressing in the tailward direction. The boundary between the dipolarized/stretched regions of the CPS thereby acts as a barrier for transport processes (Shiokawa et al., 1997). However, since we are dealing with time averaged data we cannot observe a "snapshot" of this boundary, just the resulting average dipolarization of the CPS and the decrease of the transport rates.

We also examined the occurrence of rapid flux trans- 

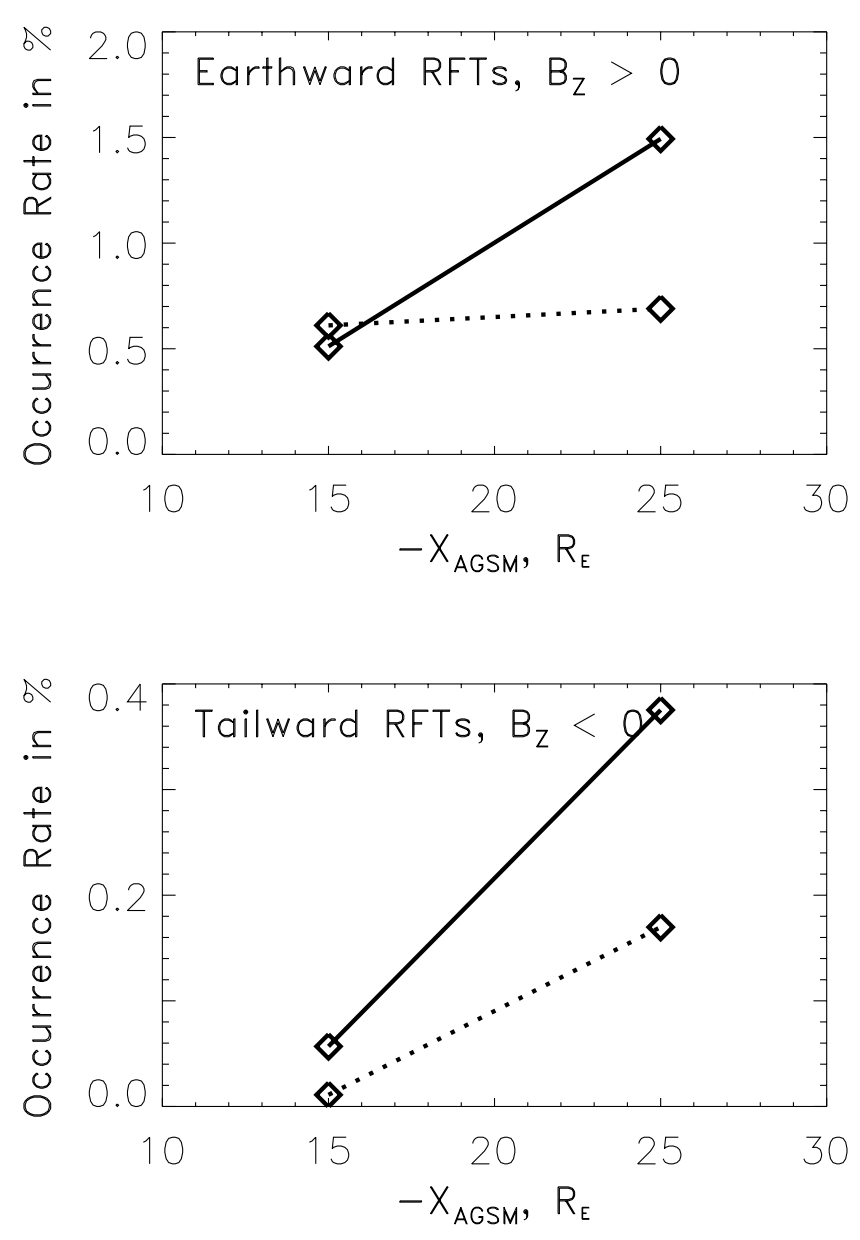

Fig. 3. Occurrence rates of earthward $\left(B_{Z}>0\right)$ and tailward $\left(B_{Z}<\right.$ $0)$ directed rapid flux transport events in the CPS. Solid lines: storm time $\left(D_{s t}<-25\right)$, dotted lines: quiet times.

port events (RFTs) during the different phases of magnetic storms. RFTs, defined by Schödel et al. (2001), are short bursts of strong flux transport in the CPS, with $E_{H}=$ $\left[\left(V_{X} B_{Z}\right)^{2}+\left(V_{Y} B_{Z}\right)^{2}\right]^{1 / 2}>2 \mathrm{mV} / \mathrm{m}$, that are directed perpendicularly to the magnetic field direction, and have very similar properties to the short bursts of fast plasma flow that are observed in the CPS (Baumjohann et al., 1990; Angelopoulos et al., 1994). Since RFTs have occurrence rates of just a few percent, we used a broader (and somewhat coarser) data base for the analysis by selecting as storm time CPS data all samples obtained at times with $D_{s t}<-25$. The resulting occurrence rates (Fig. 3) for earthward directed RFTs with a northward magnetic field show that tailward of $\sim 20 R_{E}$ they occur much more frequently during storm times than during quiet times, in agreement with the overall enhanced convection during storm times. The earthward decrease of their occurrence rate may be related to the strong pressure gradient during the magnetic storm main phase (Fig. 2), which may cause the RFTs to be braked rapidly (Shiokawa et al., 1997), such that in the 10-20 $R_{E}$ region their occurrence rate is not higher than during quiet times. Quiet time occurrence rates are $\sim 1 \%$ at all observed distance ranges, consistent with the results of Schödel et al. (2001).

Tailward RFTs with a southward magnetic field occur with significant frequency only tailward of $\sim 20 R_{E}$ consistent with the findings of Schödel et al. (2001). In fact, the occurrence rate of the tailward RFTs earthward of $\sim 25 R_{E}$ is $<0.1 \%$, which cannot be seen in Fig. 3 because of the binning. Since this type of RFT could be expected tailward of a putative near-Earth neutral line, this indicates that during the storm main phase the most earthward location of a possible NENL would be tailward of $20 R_{E}$. In this case the tailward increase of the occurrence rate of (tailward and earthward) RFTs would mean that either the NENL forms on average more frequently farther tailward than $20 R_{E}$ or that the NENL retreats tailwards during individual storm time substorms.

\section{Conclusion}

A statistical analysis of about five years of Geotail data allowed us to derive the average properties of the CPS during magnetic storms. During the recovery phase, the average state of the CPS is very similar to its state during quiet $\left(D_{s t}>-25\right)$ times, except for the ion temperature, which is still considerably increased (by $\sim 50 \%$ ). The transport of plasma and of magnetic flux is strongly increased during the storm main phase, but a steep pressure gradient apparently leads to a considerable reduction of the transport rates closer to Earth and consequently to a dipolarization of the CPS. This is reflected in the significantly increased magnetic field elevation angle in the regions $10-30 R_{E}$ during the main phase. Hence, on average, the CPS seems to inflate during the storm main phase. Increased specific ion entropy points to non-adiabatic heating processes, like a recurrent circulation of ions between the tail and the inner magnetosphere by repeated substorm activity (Liu and Rostoker, 1995). The occurrence rates of tailward rapid flux transport events point to the earthward most location of a possible near-Earth neutral line during the main phase at $\sim 20 R_{E}$.

Acknowledgements. We are grateful to S. Kokubun for generously providing the Geotail MGF data.

Topical Editor G. Chanteur thanks two referees for their help in evaluating this paper.

\section{References}

Angelopoulos, V., Kennel, C. F., Coroniti, F. V., Pellat, R., Kivelson, M. G., Walker, R. J., Russell, C. T., Baumjohann, W., Feldmann, C. W., and Gosling, J. T.: Statistical characteristics of bursty bulk flow events, J. Geophys. Res., 99, 21 257-21 280, 1994.

Baumjohann, W., Paschmann, G., and Lühr, H.: Characteristics of high-speed ion flow in the plasma sheet, J. Geophys. Res., 94, 3801-3809, 1990.

Baumjohann, W.: The near-Earth plasma sheet: An AMPTE/IRM perspective, Space Sci. Rev., 64, 141-163, 1993. 
Baumjohann, W., Kamide, Y., and Nakamura, R.: Substorms, storms, and the near-Earth tail, J. Geomagn. Geoelectr., 48, 177, 1996.

Kamide, Y., Baumjohann, W., Daglis, I. A., Gonzalez, W. D., Grande, M., Joselyn, J. A., McPherron, R. L., Phillips, J. L., Reeves, E. G. D., Rostoker, G., Shama, A. S., Singer, H. J., Tsurutani, B. T., and Vasyliunas, V. M.: Current understanding of magnetic storms: Storm-substorm relationships, J. Geophys. Res., 103, 17 705-17 728, 1998.

Kokubun, S., Yamamoto, T., Acuña, M. H., Hayashi, K., Shiokawa, K., and Kawano, H.: The Geotail magnetic field experiment, J. Geomagn. Geoelectr., 46, 7-21, 1994.

Liu, W. W. and Rostoker, G.: Energetic ring current particles generated by recurring substorm cycles, J. Geophys. Res., 100, 21 897-21 910, 1995.

Lui, A. T. Y., McEntire, R. W., and Baker, K. B.: A new insight on the cause of magnetic storms, Geophys. Res. Lett., 28, 34133416, 2001.

Mukai, T., Machida, S., Saito, Y., Hirahara, M., Terasawa, T., Kaya, N., Obara, T., Ejiri, M., and Nachida, A.: The low energy particle (LEP) experiment onboard the Geotail satellite, J. Geomagn. Geoelectr., 46, 669-692, 1994.

Schödel, R., Baumjohann, W., Nakamura, R., Sergeev, V. A., and Mukai, T.: Rapid flux tranport in the central plasma sheet, J. Geophys. Res., 106, 301-313, 2001.

Shiokawa, K., Baumjohann, W., and Haerendel, G.: Braking of high-speed flows in the near-Earth tail, Geophys. Res. Lett., 24, 1179-1182, 1997.

Siscoe, G. L.: Solar System Magnetohydrodynamics, in: SolarTerrestrial Physics, (Eds) Carovillano, R. L. and Forbes, J. M., D. Reidel Publ. Co., Dordrecht, Holland, 11-100, 1983. 